\title{
A versatile papaya mosaic virus (PapMV) vaccine platform based on sortase-mediated antigen coupling
}

\author{
Ariane Thérien ${ }^{1}$, Mikaël Bédard', Damien Carignan', Gervais Rioux', Louis Gauthier-Landry', \\ Marie-Ėve Laliberté-Gagné ${ }^{\text {, Marilène Bolduc }}{ }^{1}$, Pierre Savard ${ }^{2}$ and Denis Leclerc ${ }^{1 *}$ (1)
}

\begin{abstract}
Background: Flexuous rod-shaped nanoparticles made of the coat protein (CP) of papaya mosaic virus (PapMV) have been shown to trigger innate immunity through engagement of toll-like receptor 7 (TLR7). PapMV nanoparticles can also serve as a vaccine platform as they can increase the immune response to fused peptide antigens. Although this approach shows great potential, fusion of antigens directly to the CP open reading frame (ORF) is challenging because the fused peptides can alter the structure of the CP and its capacity to self assemble into nanoparticles-a property essential for triggering an efficient immune response to the peptide. This represents a serious limitation to the utility of this approach as fusion of small peptides only is tolerated.

Results: We have developed a novel approach in which peptides are fused directly to pre-formed PapMV nanoparticles. This approach is based on the use of a bacterial transpeptidase (sortase A; SrtA) that can attach the peptide directly to the nanoparticle. An engineered PapMV CP harbouring the SrtA recognition motif allows efficient coupling. To refine our engineering, and to predict the efficacy of coupling with SrtA, we modeled the PapMV structure based on the known structure of PapMV CP and on recent reports revealing the structure of two closely related potexviruses: pepino mosaic virus (PepMV) and bamboo mosaic virus (BaMV). We show that SrtA can allow the attachment of long peptides [Influenza M2e peptide (26 amino acids) and the HIV-1 T20 peptide (39 amino acids)] to PapMV nanoparticles. Consistent with our PapMV structural model, we show that around $30 \%$ of PapMV CP subunits in each nanoparticle can be fused to the peptide antigen. As predicted, engineered nanoparticles were capable of inducing a strong antibody response to the fused antigen. Finally, in a challenge study with influenza virus, we show that mice vaccinated with PapMV-M2e are protected from infection.
\end{abstract}

Conclusions: This technology will allow the development of vaccines harbouring long peptides containing several $\mathrm{B}$ and/or T cell epitopes that can contribute to a broad and robust protection from infection. The design can be fast, versatile and can be adapted to the development of vaccines for many infectious diseases as well as cancer vaccines.

Keywords: Papaya mosaic virus, Flexuous rod shape nanoparticles, Vaccine platform, Influenza M2e based vaccine, Sortase, Transpeptidase

\section{Background}

The use of nanoparticles as vaccine platforms is a very promising approach to improve immune response

\footnotetext{
*Correspondence: Denis.Leclerc@crchudequebec.ulaval.ca

${ }^{1}$ Department of Microbiology, Infectiology and Immunology, Infectious Disease Research Center, Laval University, 2705 Boul. Laurier, Quebec City, PQ G1V 4G2, Canada

Full list of author information is available at the end of the article
}

directed against poorly immunogenic antigens. Nanoparticles can be made of viral structural proteins that self-assemble to mimic the native organisation and conformation of a viral pathogen [1-9]. They can also present peptide antigens on their surface and induce a potent humoral response $[2-4,10]$. Being of a size similar to viruses, nanoparticles are efficiently phagocyted and processed by antigen presentation cells (APCs) such as 
dendritic cells (DC) $[11,12]$. They can also activate innate immunity through pathogen associated molecular patterns (PAMPs), which are recognized by innate receptors (pathogen recognition receptors; PRRs) [13, 14]. Therefore, the use of nanoparticles to display heterologous epitopes is a promising strategy for the development of novel vaccines.

We have developed a novel rod-shaped viral nanoparticle made of the coat protein $(\mathrm{CP})$ of papaya mosaic virus (PapMV) that is self-assembled around a ssRNA. PapMV nanoparticles trigger innate immunity efficiently [14] through engagement of toll-like receptor 7 (TLR7) [15]. After phagocytosis, nanoparticles reach the endosome of immune cells and liberate the ssRNA, which engages and activates TLR7 [15]. PapMV nanoparticles can be used as an adjuvant for improvement of vaccines [16], an immune enhancer for the treatment of cancer in immunotherapy [17], or as a vaccine platform to trigger an immune response to a specific peptide antigen [18]. Fusion of peptides directly to the open reading frame (ORF) of the PapMV CP leads to the formation of chimeric nanoparticles that can trigger either a humoral [19, 20] or a CTL response against the fused antigen [21-24]. This approach has shown the great potential of PapMV nanoparticles as a vaccine platform, but has also revealed the challenges involved in their engineering. Indeed, the fusion of peptides to the CP ORF can alter its structure and affect its capacity to self-assemble into immunogenic nanoparticles. We showed previously that the fusion of long peptides interferes with self-assembly [20].

To decrease this stress on the structure of the nanoparticles, we propose here a novel method for coupling of peptides to already self-assembled nanoparticles using the bacterial transpeptidase, sortase A (SrtA). SrtA anchors surface proteins, such as virulence factors, to the bacterial cell wall peptidoglycans of Gram-positive bacteria [25-27]. Target proteins become linked to a polyG motif at the $\mathrm{N}$-terminus of the acceptor peptidoglycan through the recognition and cleavage of their LPXTG recognition motif by SrtA. SrtA has been used widely for the site-specific labelling of proteins with a wide range of functional groups, such as fluorescent labels and green fluorescent protein (GFP) [28-31], biotin [32], PEG [29, 31,33 ], peptide nucleic acids [34], lipids [35], sugars [36], and other proteins $[30,37,38]$. A soluble form of SrtA lacking the transmembrane domain can be produced efficiently in $E$. coli and used in in vitro transpeptidation reactions.

Based on in silico modelling of the 3D structure of full length PapMV CP and of PapMV, we engineered the PapMV vaccine platform with the receptor motif of SrtA, and used SrtA to attach long peptides to the nanoparticles. This technology allows rapid and efficient coupling of peptide antigens to PapMV nanoparticles without affecting their structure. PapMV nanoparticles with SrtA-conjugated influenza M2e peptide were shown to be immunogenic, and induced protection against influenza infection.

\section{Results}

\section{Structural models of PapMV CP and PapMV}

The recent solving of the near-atomic structure of Bamboo mosaic virus (BaMV) [39] and Pepino mosaic virus (PepMV) [40] by cryo-electron microscopy (cryo-EM), has allowed the structural details of Potexvirus viruses to be revealed at an atomic level. Interestingly, the CPs of these two Potexviruses share high structural conservation with the structure of the truncated $\mathrm{CP}$ of PapMV (PDB ID 4DOX) [41] with root mean square deviations (RMSD) of around 1.6 and $3 \AA$ for the backbone heavy atoms of PepMV (PDB ID 5FN1) and BaMV (PDB ID 52AT), respectively (Fig. 1a). Moreover, previous low resolution cryo-EM data reported by our group on PapMV [41] demonstrates that it adopts a capsid symmetry similar to that adopted by BaMV and PapMV, consisting of a left-handed helix of $\sim 130 \AA$ in diameter, with $\sim 10 \mathrm{CP}$ subunits per turn and a pitch of $\sim 35 \AA$. These structural similarities prompted us to elaborate a structural model for PapMV based on BaMV and PapMV to guide the development of our nanoparticles following a rational design approach. Briefly, a homology model of the complete PapMV CP based on the structure of the truncated PapMV CP (PDB ID 4DOX), BaMV CP and PepMV $\mathrm{CP}$ was generated using I-TASSER [42]. The complete PapMV CP was then aligned on the subunit CPs of PepMV (PDB 5FN1) to construct the model (Fig. 1b). The viral ssRNA (in orange) was positioned by homology with PepMV (Fig. 1b, c).

As observed for the structures of BaMV and PepMV, the N-terminal ends of PapMV CPs are exposed on the surface of the virus, while the C-terminii are located in the central cavity (Fig. 1c). Therefore, fusions to the $\mathrm{N}$-terminus can modify the surface of PapMV nanoparticles and affect the interface of interaction between nanoparticles and immune cells. Fusions made at the C-terminus could cause steric hindrance in the central cavity, interfering with nanoparticle assembly, as indicated by the cross-section view of the PapMV nanoparticle model displayed in Fig. 1c (right illustration) and as previously reported [20]. Considering that the C-terminal sections of two CPs separated by one capsid turn define a cavity of around $25 \AA$ (Fig. 1c), it is anticipated that the longest $\mathrm{C}$-terminal fusion allowed in the core of the nanoparticles would be $\sim 17$ amino acids if arranged as an $\alpha$-helix. But, fusion of long peptides on each side of the nanoparticles where the $\mathrm{C}$-terminus are accessible 

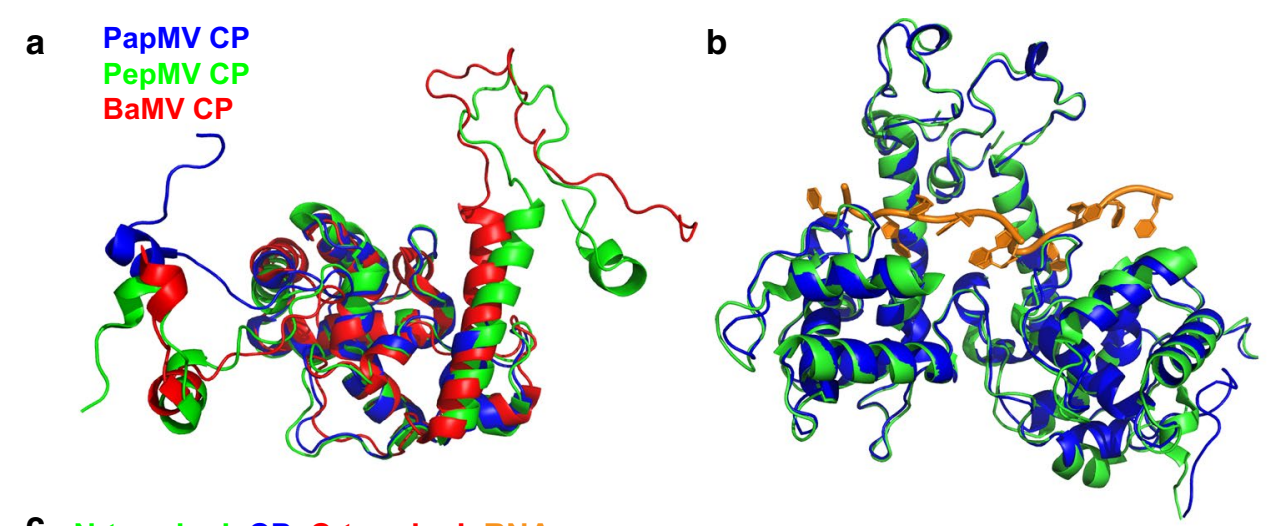

C N-terminal CP C-terminal RNA
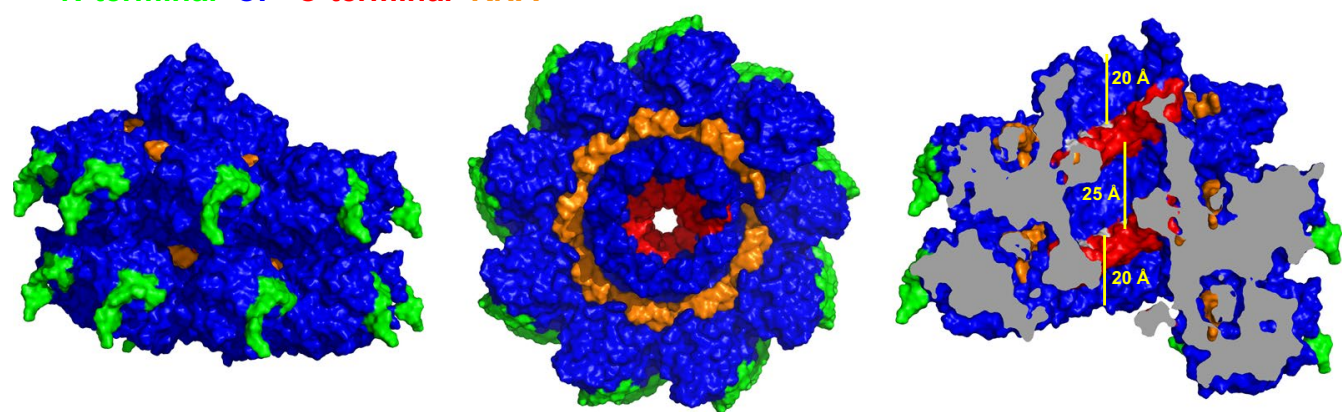

Fig. 1 Modelling of the PapMV CP and PapMV structure. a The full-length structure of the PapMV CP was modelled based on the recently published structure of two members of the potexvirus group: BaMV CP and PepMV CP. The core region of PapMV CP (PDB 4DOX, blue) superimposes well on the core region of PepMV CP (PDB 5FN1, green) and BaMV CP (PDB 5A2T, red). b Superposition of two subunits of the PapMV model (blue) with two CP subunits of the PepMV structure (green, PDB 5FN1) demonstrates the concordance between the two structures. c To show how each of the PapMV CP interacts with each other, and with the ssRNA in the nanoparticle, we modeled the self assembly of the 18 subunits ( 2 turns) that comprise PapMV nanoparticles. The CP N-terminal residues (10 first) are shown in green, the CP core in blue, the CP C-terminus in red (10 last residues), and the RNA is in orange. The last C-terminal residue of the CP is displayed in light grey in the cutaway view on the right, and the bars represent $20 \AA$ - the distance separating CP C-terminal residues from the PapMV nanoparticle exterior at both extremities, and $32 \AA$-the distance between two CP C-terminal residues separated by one capsid turn

is possible without changing the surface of the nanoparticle. Therefore, it suggests that the development of a method allowing peptide coupling to available C-terminus at the extremity of already-assembled nanoparticles is an attractive approach for the coupling of long peptides to our vaccine platform.

\section{Engineering of the PapMV nanoparticles with the SrtA recognition motif}

Based on the structural model of the PapMV nanoparticle presented in Fig. 1c, we chose to fuse the sortase A (SrtA) LPETGG recognition motif followed by a $6 \times \mathrm{H}$ tag to the C-terminus of the PapMV CP. This fusion is preceded by the linker TSTTR, which was introduced to allow the LPETGG motif to reach the exterior of the nanoparticles at both ends (Fig. 1c-right panel), making it available for the transpeptidation reaction. Indeed, assuming a maximal length of $3.5 \AA$ per amino acid, the PapMV structural model suggests that around 5 amino acids are required to span the $20 \AA$ distance separating
CP C-terminal residues from the nanoparticle exterior at both extremities (Fig. 1c-right panel). Fusion of the receptor motif did not alter the capacity of the CP to self-assemble around the ssRNA, leading to generation of nanoparticles (PapMV-SrtA) with an average length of $40 \mathrm{~nm}$ vs $54 \mathrm{~nm}$ for the WT PapMV nanoparticle, as shown by dynamic light scattering (DLS) (Fig. 2a). The width of the DLS curve in the two types of nanoparticles was comparable, suggesting that nanoparticles are of different lengths ranging from $\sim 20$ to $\sim 100 \mathrm{~nm}$. Electron microscopy (EM) confirmed that PapMV-WT and PapMV-SrtA are similar in appearance (Fig. 2b). As a control, we engineered another PapMV CP lacking the TSTTR linker preceding the SrtA recognition motif [PapMV-SrtA (short)] (Additional file 1: Figure S1). This construct is predicted to not permit coupling because the SrtA recognition motif is hidden too far within the core cavity of the PapMV nanoparticle. As with PapMV-SrtA, the fusion did not interfere with self-assembly, and generated nanoparticles comparable to those of WT PapMV, 


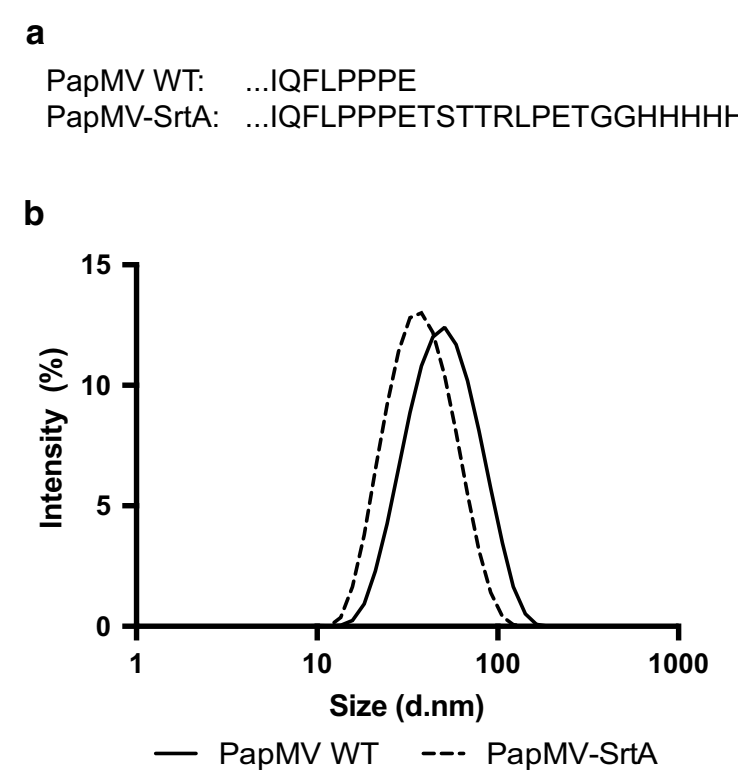

C
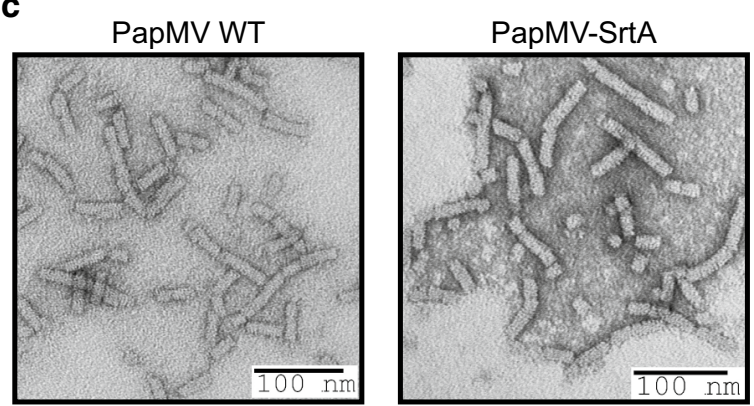

Fig. 2 Engineering of PapMV coat protein carrying the SrtA recognition motif. a Amino acid sequence of the PapMV CP C-terminus of the WT CP as compared to the engineered PapMV CP that harbours a linker of 5 amino acids (TSTTR) followed by the SrtA recongnition motif (LPETGG); a $6 \times$ His tag was inserted at the C-terminus of the PapMV coat protein (named PapMV-SrtA). b The length of the rod-shaped nanoparticles was assessed by dynamic light scattering (DLS). PapMV-SrtA (40 nm) was showed to be slightly smaller than the WT PapMV (54 nm). c Transmission electron microscopy (TEM) of WT PapMV (left) and PapMV-SrtA (right) nanoparticles shows the rod shape of the nanoparticles

as confirmed by DLS (Additional file 1: Figure S1B) and electron microscopy (Additional file 1: Figure S1C).

\section{SrtA-mediated coupling of peptides onto PapMV nanoparticles}

To achieve SrtA-mediated coupling onto PapMV nanoparticles, we chose two long peptides of 26 amino acids (influenza M2e peptide) and 39 amino acids (HIV-1 T20 peptide), respectively. The peptide M2e is derived from the extracellular domain of the matrix protein 2 (M2e) of influenza virus. M2e is highly conserved in most influenza A strains [43] and is a valuable antigen in inducing protection to influenza infection $[19,44]$. The T20 peptide is derived from the surface glycoprotein gp41 of human immunodeficiency virus (HIV-1) [45]. A monoclonal antibody (2F5) directed towards this peptide was shown to neutralise HIV-1 infection in vitro, and has been proposed for use as a vaccine antigen [46].

We performed transpeptidation reactions using $25 \mu \mathrm{M}$ of PapMV-SrtA nanoparticles (based on the amount of PapMV CP evaluated by BCA) incubated with $50 \mu \mathrm{M}$ of either the M2e or T20 peptide and $50 \mu \mathrm{M}$ of SrtA. Following the coupling reaction, the SrtA and the free peptides were removed by filtration, and the samples were loaded on SDS-PAGE to reveal the coupling efficiency (Fig. 3a-top panel). PapMV CP coupled to either the M2e or the T20 peptide migrated as slightly higher molecular weight proteins than the uncoupled PapMV-SrtA CP on SDS-PAGE (Fig. 3a). The identity of the higher band was further confirmed by immunoblotting using specific antibodies directed towards PapMV CP (Fig. 3a-second panel), or the M2e peptide (Fig. 3a-fourth panel). Also, we used a specific antibody directed to the $6 \times \mathrm{H}$ tag that, as expected, reacted only with the uncoupled PapMV-SrtA CP (Fig. 3a-third panel). To assess the coupling efficacy precisely, we calculated the ratio between the intensity of the coupled $\mathrm{CP}$ and that of the total $\mathrm{CP}$ (coupled + uncoupled) on SDS-PAGE (Fig. 3a), which revealed a coupling efficacy of $35 \%$ with the M2e peptide and $32 \%$ with the T20 peptide. We performed six coupling reactions and always obtained a similar coupling efficiency, which ranged from 26 to $38 \%$ for M2e and 30 to $34 \%$ for the T20 peptide (not shown). To evaluate whether the structure of the nanoparticles was affected by the coupling reaction, we compared the length of the PapMV-SrtA nanoparticles with those conjugated with M2e or T20 peptides (Fig. 3b). The nanoparticles were similar in length compared to PapMV-SrtA, as shown by DLS (Fig. 3b), and in their appearance in EM (Fig. 3c).

As expected from our prediction based on the structure of the PapMV-SrtA CP, only a fraction (about 1/3) of the CP reacted with SrtA. We observed that coupling was saturated with $50 \mu \mathrm{M}$ of peptide and no additional coupling was observed when 100 or $200 \mu \mathrm{M}$ was used (Additional file 2: Figure S2). Finally, we confirmed that coupling reactions with the PapMV-SrtA(short) (Additional file 3: Figure S3A) were very inefficient as compared to those with PapMV-SrtA, which shows the importance of the linker (TSTTR) in making the SrtA recognition motif solvent-accessible (Additional file 3: Figure S3B). Coupling on PapMV-SrtA(short) nanoparticles was consistently ineffective (four repeated experiments), unlike coupling on the PapMV-SrtA construct as revealed by immunoblot using an antibody directed against the M2e peptide (Additional file 3: Figure S3B). 


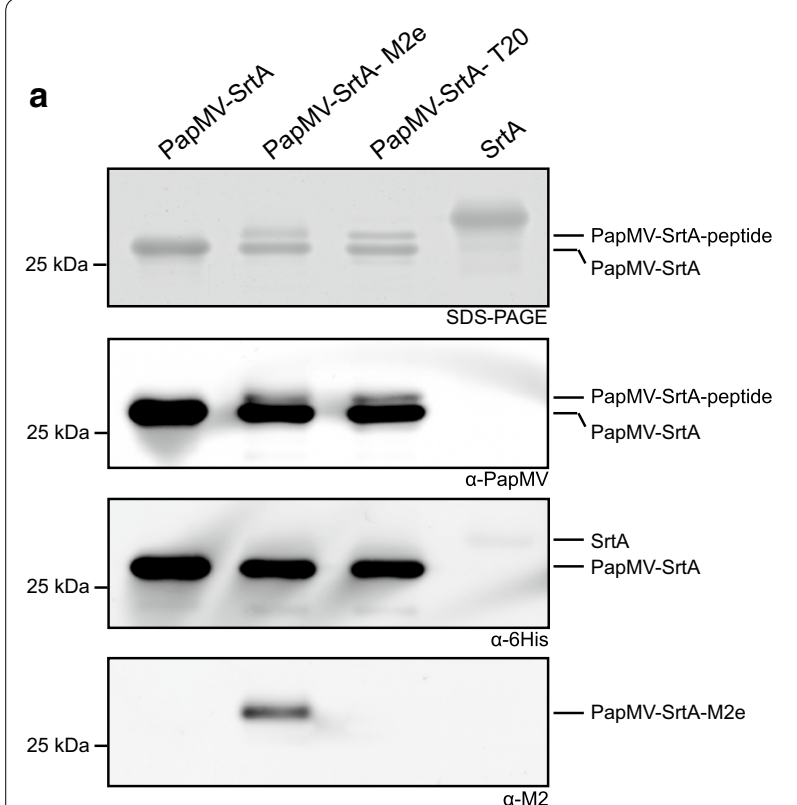

C

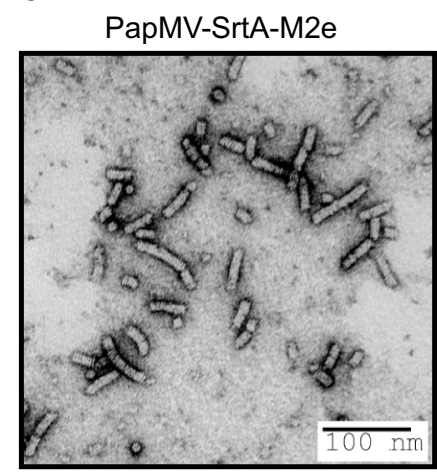

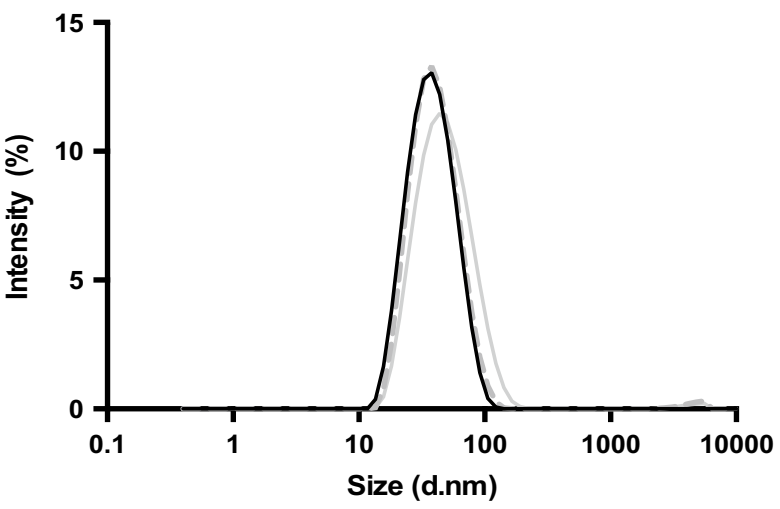

- PapMV-SrtA - - PapMV-SrtA-M2e — PapMV-SrtA-T20

Fig. 3 Coupling of T2O and M2e onto PapMV-SrtA nanoparticles. a To reveal the efficacy of coupling of peptides onto the PapMV vaccine platform, we performed a 5\% Tris-Glycine SDS-PAGE. The coupling induced by the sortase is covalent, and induced a shift on the gel as compared with the WT PapMV CP. Western blot of SrtA-conjugated nanoparticles PapMV-SrtA-M2e and PapMV-SrtA-T20 revealed fusion of the peptide on the PapMV CP. Western blots were directed against the PapMV CP, the $6 \times \mathrm{H}$ tag, or the M2e peptide, as indicated under each panel. To evaluate if coupling of the peptide affected the structure of the nanoparticles, we evaluated their size by DLS analysis (b), comparing PapMV-SrtA (54 nm) to conjugated PapMV-SrtA-M2e $(43 \mathrm{~nm})$ and PapMV-SrtA-T20 $(52 \mathrm{~nm})$ nanoparticles. We also used TEM of PapMV-SrtA-M2e (left) and PapMV-SrtA-T20 (right) nanoparticles to confirm that the rod shape was preserved after the coupling experiment

\section{The PapMV-SrtA vaccine platform enhances the humoral response to the T20 peptide}

To assess the capacity of the PapMV-SrtA vaccine platform to improve the humoral response to the T20 peptide, we immunized Balb/C mice (5/group) twice, with a 14-day interval, with $90 \mu \mathrm{g}$ of PapMV-SrtA, 30 or $90 \mu \mathrm{g}$ of PapMV-SrtA-T20, or $90 \mu \mathrm{g}$ of PapMV-SrtA with $40 \mu \mathrm{g}$ of free T20 peptide. Blood levels of total anti-T20 IgG and IgG2a were assessed by ELISA 13 days after each immunization (Fig. 4a-d). Mice vaccinated with PapMV-SrtA or PapMV-SrtA and the free T20 peptide did not develop any detectable T20 immunoglobulins. However, $30 \mu \mathrm{g}$ of PapMV-SrtA-T20 was sufficient to mount a robust antibody response against the T20 peptide after a single immunization (Fig. 4ab). This result confirms that coupling of the peptide to the PapMV vaccine platform improved the immunogenicity of the peptide significantly. We also observed a rapid class switch towards a $\mathrm{TH} 1$ response, since a strong IgG2a response specific to the T20 peptide was recorded after the first immunization (Fig. 4b), which is expected with the PapMV vaccine platform as previously reported $[19,44,47,48]$. The IgG2a isotype is known to show a higher avidity for the antigen, and is therefore more efficient for neutralization 
a

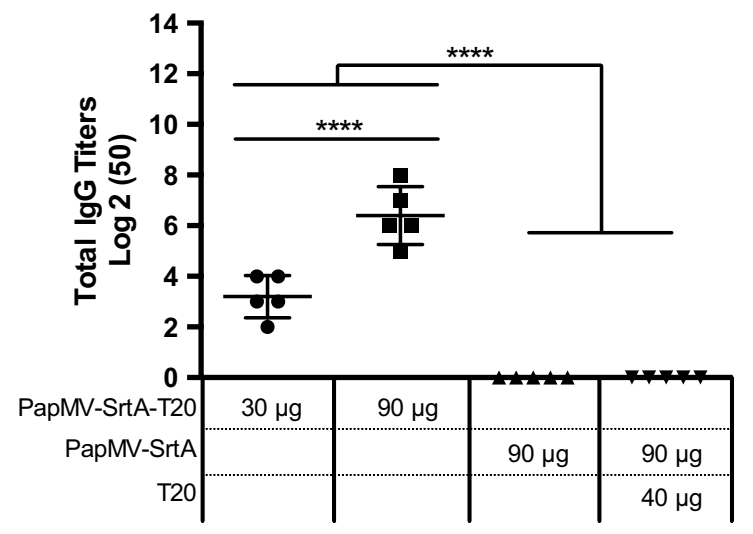

C

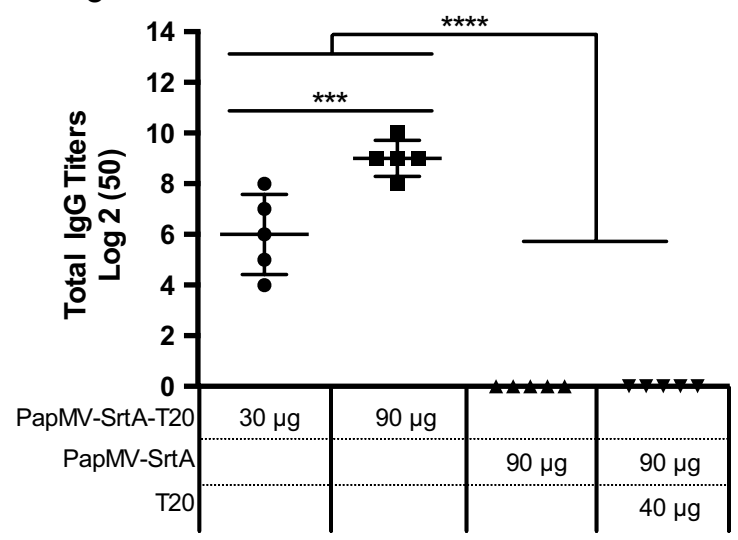

b

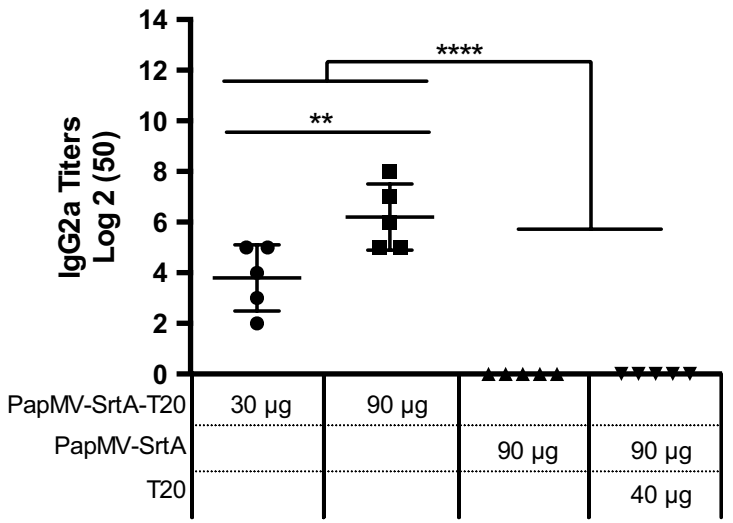

d

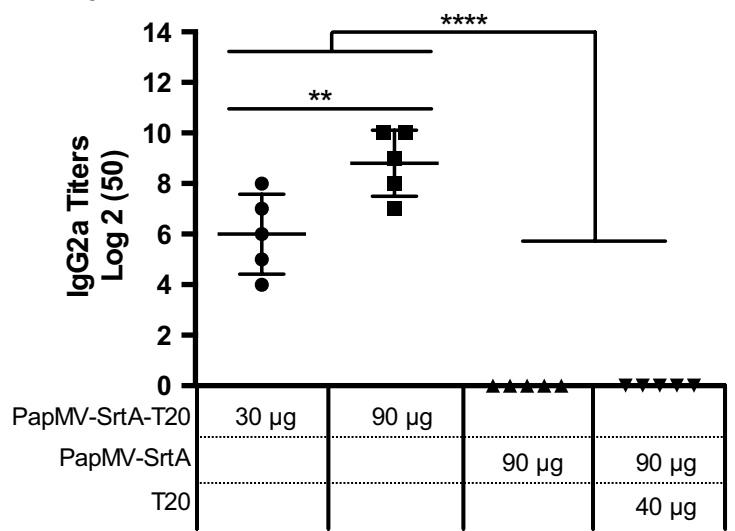

Fig. 4 PapMV-SrtA-T20 induces a T20 specific antibody response. We immunized animals [Female Balb/C mice (5/group)] twice, with a 14-day interval by the intramuscular route with either 30 or $90 \mu \mathrm{g}$ of PapMV-SrtA-T20, PapMV-SrtA or T20 peptide, to evaluate if coupling of T20 peptides fused to the surface of PapMV nanoparticles enhance the humoral response to the T20 peptide antigen. Blood was taken 14 days following each immunization, and ELISA assays were performed to evaluate levels of anti-T20 total lgG (a) or lgG2a (b) after the first immunization, and anti-T20 total lgG (c) or IgG2A (d) after the booster immunization. ${ }^{* * *} P<0.0001$ all PapMV-SrtA-T20 groups against PapMV-SrtA alone, or PapMV-SrtA and T20 peptide. ${ }^{* *} P<0.001$ between 30 and $90 \mu$ g of PapMV-SrtA-T20 total lgG (d27), ${ }^{* * *} P<0.0001$ total $\lg G(\mathrm{~d} 13)$ and ${ }^{* *} P<0.01 \lg$ G2a (d13 and d27)

of a viral infection. Finally, and as expected, the boost immunization raised the total IgG and IgG2a titers significantly (Fig. 4c, d).

\section{The PapMV vaccine platform enhances the humoral response to the $M 2$ e peptide and induces protection to an influenza challenge}

To evaluate the efficacy of our approach in an animal infectious model, we immunized mice with PapMVSrtA-M2e, and evaluated the humoral response directed towards the M2e peptide, and the capacity to provide protection to an influenza challenge. We also included in this study, as a reference, the PapMV-sM2e construct previously generated in our laboratory [19]. PapMVsM2e harbours a fusion of a 9-amino-acid long peptide (EVETPIRNE) corresponding to the central region of the M2e peptide. In these nanoparticles, every PapMV CP molecule harbours a fusion of the small M2e peptide. This construct was previously shown to induce production of antibodies to the full length M2e peptide, and to provide protection to an influenza challenge [19]. To compare the efficacy of the two constructs, five mice per group received prime and booster immunizations at intervals of 14 days with either 30 or $90 \mu \mathrm{g}$ of PapMVSrtA-M2e, $30 \mu \mathrm{g}$ PapMVCP-sM2e or formulation buffer. Thirteen days following the first and the second immunization, sera were collected, and levels of total IgG and IgG2a directed against the M2e peptide were measured by ELISA. Interestingly, the levels of antibodies directed against the M2e (total IgG and IgG2a) were similar between all groups following the first immunization (Additional file 4: Figure S4). After two immunizations, total IgG and IgG2a were comparable between groups immunized with $30 \mu \mathrm{g}$ of PapMV-SrtA-M2e or $30 \mu \mathrm{g}$ of 
PapMV-sM2e (Fig. 5a, b). However, immunization with $90 \mu \mathrm{g}$ of PapMV-SrtA-M2e triggered a humoral response against the M2e peptide that was significantly higher than that of $30 \mu \mathrm{g}$ of either PapMV-SrtA-M2e or PapMVsM2e (Fig. 5a).

To evaluate the capacity of the candidate vaccine to protect against influenza, we challenged immunized mice 14 days after the second immunization with $1 \times$ LD80 of influenza $\mathrm{A} / \mathrm{WSN} / 33$ virus. This experiment was critical because it allowed us to demonstrate that the nanoparticle vaccine platform not only improves the humoral response to a peptide antigen, but also induces production of antibodies that protect the animal against an influenza infection. After the challenge, three different assessments were used to confirm the efficacy of the vaccine formulation: (1) weight loss, which gives a reliable readout because infected mice loose weight rapidly following an influenza challenge. Because weight can be evaluated precisely each day following the challenge, it becomes an important readout to evaluate the efficacy of the protection; (2) assessment of symptoms, which is an empirical measurement that usually correlates with weight loss. Symptoms were assessed by technicians blind to treatment group to decrease the risk of assessment bias; (3) survival, which is the ultimate proof of protection induced by a vaccine formulation. To comply with the animal ethics protocol of our institution, we sacrificed animals that lost more than $20 \%$ of their body weight.

In brief, mice immunized with PapMV-SrtA-M2e (30 and $90 \mu \mathrm{g})$ and PapMV-sM2e $(30 \mu \mathrm{g})$ showed minimal weight loss as compared to mice immunized with formulation buffer (Fig. 5c). Clinical signs of infection (ruffled fur, curved back, mobility loss) were significantly reduced in mice immunized with $90 \mu \mathrm{g}$ of PapMV-SrtAM2e, although weight loss was not significantly different between mice immunized with PapMV-SrtA-M2e or PapMV-sM2e (Fig. 5c, d). Formulation buffer immunized mice showed severe clinical symptoms and significant weight loss, reaching a mean of $79.3 \%$ of their initial weight, at day 8 post-infection. Only groups immunized with $90 \mu \mathrm{g}$ of PapMV-SrtA-M2e or with PapMV-sM2e showed $100 \%$ survival. While $60 \%$ of mice immunized with $30 \mu \mathrm{g}$ of PapMV-SrtA-M2e survived the infection, only $20 \%$ of formulation buffer immunized mice survived (Fig. 5e). To confirm this result, we repeated the same experiment with 10 mice per group, evaluated the humoral response after two immunizations (Additional file 5: Figure S5AB), and performed the challenge with 1LD80 of the WSN/33 influenza strain. We monitored weight loss (Additional file 5: Figure S5C), symptoms (Additional file 5: Figure S5D) and survival (Additional file 5: Figure S5E). Consistent with the previous experiment (Fig. 5), the two best performing vaccine formulations were PapMV-sM2e and PapMVSrtA-M2e, which induced a significant protection to influenza challenge.

\section{Discussion}

In this study, we built a 3D model of PapMV CP and PapMV nanoparticles based on the recently published structures of two other members of the potexvirus family: BaMV [39] and PepMV [40]. This model was used to refine engineering of the PapMV vaccine platform. We confirmed experimentally that a fusion of a 17 amino acid peptide harbouring the SrtA recognition motif to the $\mathrm{C}$-terminus of the CP was tolerated, and did not interfere with self-assembly. As anticipated from our PapMV structure prediction, only a fraction of $\mathrm{CP}$ subunits in the nanoparticle seem to present the SrtA recognition motif to the sortase, leading to coupling efficiencies that reached a plateau at around 30\% for both peptides tested. Coupling probably occurs on the extremities of the nanoparticles, leaving the surface free for interactions with immune cells. In fact, even if the reaction was pushed by increasing the amount of peptide, the amount of coupling did not increase, suggesting that we had reached saturation of all available sites. According to the structural model, the C-termini of the $\mathrm{CP}$ units are located in the interior cavity of the nanoparticle, and are thus not available for the coupling reaction. The coupling of peptide antigens to already pre-assembled nanoparticles allowed attachment of longer peptides (26 and 39 amino acids) without affecting the structure of the nanoparticle. Consistent with our model, fusion of a 24-amino-acid peptide to the CP C-terminus led to the formation of unstable nanoparticles that were less immunogenic [20]. With the SrtA approach, we can fuse longer peptides containing multiple epitopes-a major advantage when developing new vaccines.

While SrtA has been widely used for the modification and conjugation of diverse proteins including antibodies, protein-protein, and protein-to-solid-support conjugates, few studies have investigated the modification of nanoparticles by SrtA-mediated conjugation. A recent study showed that SrtA could be used to link FITC or GFP to the N-terminus of cowpea chlorotic mottle virus (CCMV) coat protein expressing an $\mathrm{N}$-terminal glycine residue [28]. Although the authors of the latter study did not use SrtA to functionalize the coat proteins postassembly, they were able to co-assemble conjugated and unconjugated CCMV into VLPs to encapsulate the cargo. In another study, M13 bacteriophage surface proteins were modified using SrtA from Staphylococcus aureus and Streptococcus pyogenes [49]. Various proteins and small molecules have been attached to either the pIII, 


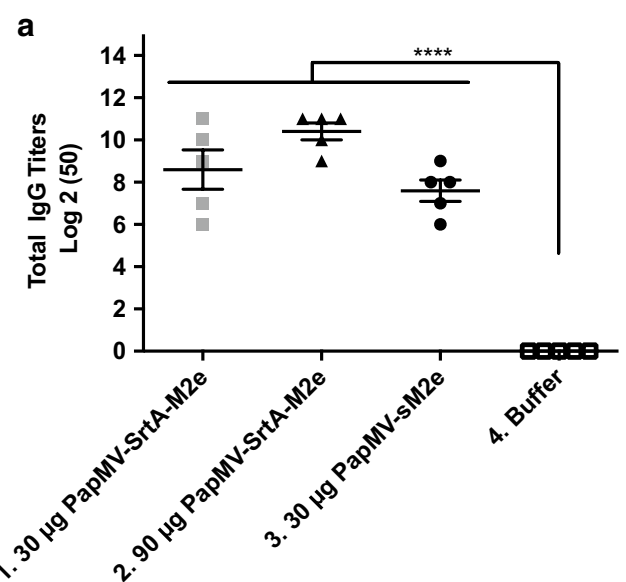

C

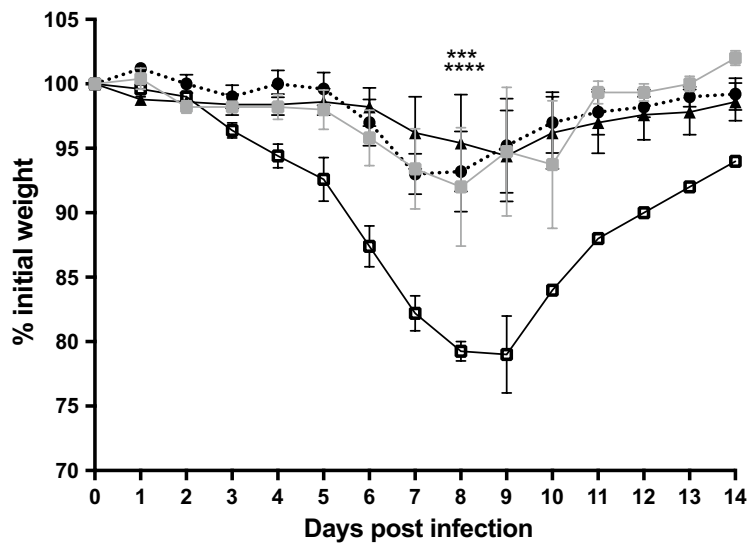

b

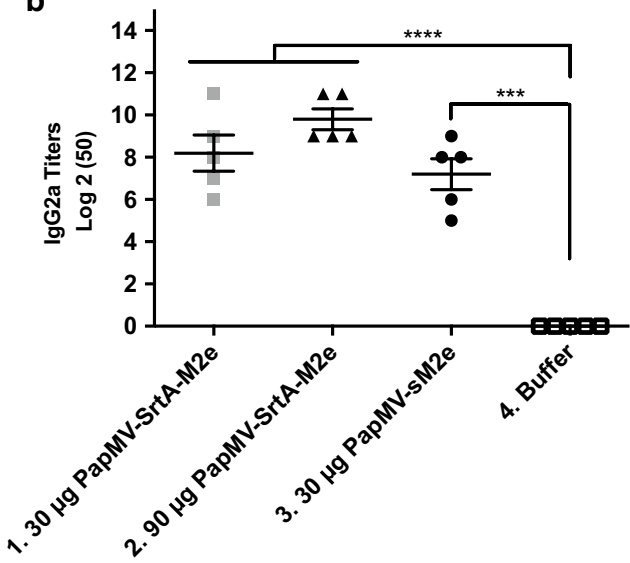

d

e
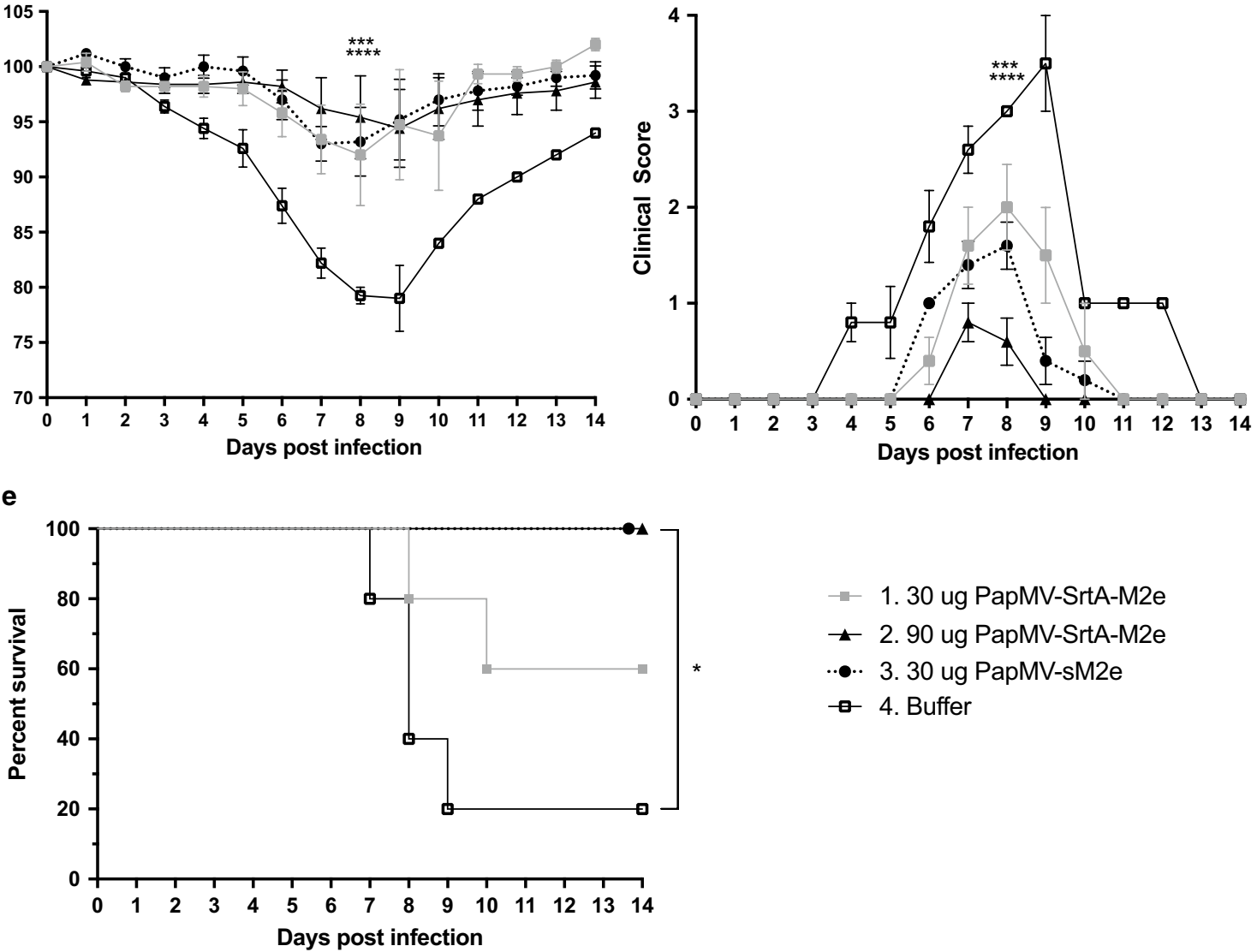

- 1. 30 ug PapMV-SrtA-M2e

— 2.90 ug PapMV-SrtA-M2e

-. 3. 30 ug PapMV-sM2e

- 4. Buffer

Fig. 5 PapMV-SrtA-M2e induces a specific anti-M2e immune response and protection against an influenza challenge. The M2e peptide has been previously shown to be a highly conserved epitope of influenza A virus that is able to induce protection against an influenza challenge. Therefore, Female Balb/C mice (5 per group) were immunized twice with PapMV nanoparticles coupled to the M2e peptide using the sortase (PapMV-SrtAM2e), PapMV-sM2e [where a small version of the M2e (sM2e) is fused directly to the N-terminus of the PapMV CP] (positive control), or buffer of the vaccine formulation alone. Blood was taken 13 days following the last immunization, and ELISA assays performed to evaluate levels of anti-M2e total lgG (a) or lgG2a (b). ${ }^{*} \mathrm{P}<0.01$ for groups 2 vs 3 total lgG titers and ${ }^{*} \mathrm{P}<0.05$ for group 2 vs 3 lgG 2 a titers. To assess the capacity of the PapMVSrtA-M2e nanoparticles to induce protection to an influenza challenge, immunized mice were infected with $1 \times$ LD80 of influenza A/WSN/33 virus 14 days after the last immunization, and followed for clinical symptoms and survival for 14 days. c Mean clinical score of infection signs on a scale of 0 to 4 . ${ }^{* * *} P<0.001$ for group 1 vs 4 , and for group 2 vs 3 , ${ }^{* * * *} P<0.0001$ for groups $2-3$ vs 4 , and for group 1 vs 2 , all at day 8 post-challenge. d Mean weight loss expressed as percentage of initial weight. ${ }^{* *} P<0.001$ for group $1 \mathrm{vs} 4$, and ${ }^{* * *} P<0.0001$ for group $2-3$ vs 4 . e Survival of mice expressed as Kaplan-Meier survival curves. ${ }^{*} P<0.5$ for groups $2-3$ vs 4 
pIX or pVIII bacteriophage proteins expressing N-terminal glycines but the potential of this approach as a vaccine platform has not been tested through immunization of animals. Both these studies used SrtA to modify the $\mathrm{N}$-terminus of the $\mathrm{CP}$, while, in contrast, our system conjugates peptides to the $\mathrm{C}$-terminus of the $\mathrm{CP}$ to minimise any modification of the nanoparticle surface that may interfere with the interaction with immune cells. Finally, fusion of the SrtA recognition motif to an internal surface-exposed loop of the hepatitis B virus (HBV) core protein led to efficient conjugation of a peptide derived from the enterovirus 71 (EV71) and the AD-4 domain on glycoprotein $\mathrm{B}(\mathrm{gB})$ of human cytomegalovirus (HCMV) on HBV icosahedral nanoparticles [50]. These SrtA-modified HBV nanoparticles improved the immune responses directed towards their antigen.

PapMV nanoparticles engineered with the SrtA recognition motif were shown to significantly improve the humoral response triggered towards the attached peptides. Although only one-third of the CPs in PapMVSrtA nanoparticles became coupled to the M2e or T20 peptide, a robust immune response was triggered and PapMV-SrtA-M2e was capable of protecting mice from an influenza challenge. The main differences between the PapMV vaccine platform and the HBV nanoparticle are the shape of the particle and their differential ability to trigger innate immunity: HBV is icosahedral whereas PapMV is rod-shaped. Furthermore, PapMV has the unique capacity to trigger innate immunity through the engagement of TLR7, which results in the induction of IFN- $\alpha$ secretion $[15,17]-a$ major advantage for the development of an efficient vaccine. The PapMV vaccine platform is also capable of inducing a class switch toward production of IgG2a antibodies, which implies cooperation of CD4+ immune cells [51]. IgG2a antibodies control viral infection better than other immunoglobulins due to their higher avidity and their capacity to trigger the antibody-dependent cell-mediated toxicity (ADCC) that is critical to eliminate virus-infected cells with natural killer (NK) cells [52].

Considering the speed at which a candidate vaccine can be developed, and the versatility of this approach, we believe that our SrtA method is advantageous for the presentation of antigens on the PapMV vaccine platform. Sometimes an antigen alone is sufficient to provide protection but, in most cases, this is a very limiting factor.

\section{Conclusion}

The covalent attachment of antigens onto PapMV nanoparticles using SrtA allows fusion of long peptides (26-39 amino acids) to a vaccine platform. The covalent linkage of the antigen to the platform enhanced significantly the immune response directed to the antigens, and provided protection to challenge with a viral infection (influenza). This approach is more versatile than fusion of the antigen directly to the ORF of the PapMV CP-a route that tolerates fusion of only small peptides. Fusion of longer peptides to the ORF can impair CP structure and its capacity to self-assemble into nanoparticles, and formation of nanoparticles is essential to obtain a strong immune response. Thus, the SrtA-based tagging of peptide antigens onto already pre-formed PapMV nanoparticles allows for fast, flexible and easy nanoparticle modification, representing a promising tool in vaccine design against infectious diseases, as well as in the development of vaccines in cancer immunotherapy or tailormade vaccine candidates for personalised medicine [17].

\section{Methods}

Production and purification of PapMV nanoparticles

The PapMV nanoparticles harbouring the C-terminal LPETGG SrtA recognition site with (PapMV-SrtA) or without (PapMV-StrA9short) the TSTTR linker, and PapMV-M2e and PapMV nanoparticles were kindly provided by Folia Biotech (Quebec City, Quebec, Canada). The nanoparticles were produced and purified as described elsewhere [19]. Briefly, PapMV CPs were produced in E. coli. Purified CPs were assembled in vitro with a non-coding synthetic ssRNA derived from the native sequence of papaya mosaic virus RNA. Levels of contaminant LPS were measured by a Limulus Amebocyte Lysate assay (LAL) (Lonza, Walkersville, Maryland, USA). The LPS concentration in the final nanoparticle samples was always below 50 endotoxin units (EU)/mg of protein, and was thus considered negligible. The amount of nanoparticles used to perform each experiment was estimated using the BCA method (Thermo ScientificTM PierceTM BCA Protein Assay), a detergent-compatible formulation based on bicinchoninic acid (BCA) for the colorimetric detection and quantitation of total protein. Since PapMV CP is the main component of the nanoparticles ( $>95 \%$ of the total nanoparticle weight), it is a reliable method to quantify the amount of nanoparticles used in the experiments conducted in this study.

\section{Expression and purification of recombinant sortase $\mathrm{A}$}

Expression and purification of recombinant sortase A followed the method described in [53], with the following modifications: (1) $2 \times \mathrm{YT}$ (16 g bacto-tryptone, $10 \mathrm{~g}$ yeast extract, $5 \mathrm{~g} \mathrm{NaCl}, \mathrm{pH} 7.0)$ culture medium was used instead of LB medium, (2) IPTG induction was carried out when culture $\mathrm{OD}_{600 \mathrm{~nm}}$ reached $1.0 \pm 0.1$, (3) E. coli was harvested by one 15 -min centrifugation at $9000 \times g$ at $4{ }^{\circ} \mathrm{C}$, (4) bacterial pellet from $1 \mathrm{~L}$ of culture was suspended in $40 \mathrm{~mL}$ of cold lysis buffer $(50 \mathrm{mM}$ Tris $-\mathrm{HCl}$ $\mathrm{pH}$ 7.5, $500 \mathrm{mM} \mathrm{NaCl}, 10 \mathrm{mM}$ imidazole), (5) bacteria 
were lysed by three passages through an Emulsiflex C5 homogenizer (Avestin, Ottawa, Ontario, Canada) at 15,000-25,000 psi, (6) $100 \mathrm{U}$ of benzonase (SigmaAldrich Inc. Saint-Louis, Missouri, USA) and $5 \mathrm{mM}$ of $\mathrm{MgCl}_{2}$ were added to the cell lysate and incubated for 20 min with gentle agitation before cell lysate clarification by centrifugation at $20,442 \times g$ for $20 \mathrm{~min}$, (7) the clarified supernatant was applied to a $40 \mathrm{~mL}$ IMAC column packed with Ni Sepharose 6 Fast Flow Resin (GE Healthcare Life Sciences, Little Chalfont, Buckinghamshire, United Kingdom), and washed with 10 column volumes (CV) of lysis buffer and $5 \mathrm{CV}$ of wash 2 buffer $(50 \mathrm{mM}$ Tris- $\mathrm{HCl} \mathrm{pH} 7.5,150 \mathrm{mM} \mathrm{NaCl}, 25 \mathrm{mM}$ imidazole). Purification on the IMAC column was done with a ÄKTA purifier 10 FPLC (GE Healthcare Life Sciences). Imidazole was removed using a Sartoflow(R) Slice 200 benchtop crossflow system for diafiltration with a $10 \mathrm{kDa}$ cutoff (Sartorius, Göttingen, Germany). LPS contaminants were removed on an ion exchange membrane bound to quaternary ammonium ligands using the ÄKTA purifier 10, and measured by the LAL assay (Lonza, Basel, Switzerland). LPS levels were under $50 \mathrm{EU} / \mathrm{mg}$ of protein and were considered negligible. The yield of SrtA was approximately $100 \mathrm{mg} / \mathrm{L}$ of culture.

\section{Peptides}

Synthetic peptides, with over 90\% purity, used for SrtA labelling, GGG-M2e (GGGSLLTEVETPIRNEWGCRCN DSSD) and GGG-T20 (GGGYTSLIHSLIEESQNQQ EKN EQELLELDKWASLWNWF) were purchased from GenScript (United States) and Biomatik (Canada), respectively.

\section{Evaluation of the structural properties of nanoparticles by dynamic light scattering and transmission electron microscopy}

Nanoparticle size was determined by dynamic light scattering (DLS) with a ZetaSizer Nano ZS (Malvern, Malvern, Worcestershire, United Kingdom). PapMV nanoparticles were diluted to $0.12 \mathrm{mg} / \mathrm{mL}$ in $10 \mathrm{mM}$ Tris$\mathrm{HCl}, \mathrm{pH}$ 8.0, and loaded into a disposable plastic cuvette. Samples were measured four times, and average results generated by cumulative analysis.

Nanoparticle shape was assessed by transmission electron microscopy (TEM). Samples were diluted to $0.02 \mathrm{mg} / \mathrm{mL}$ and mixed at a $1: 1$ ratio with a $3 \%$ solution of uranyl acetate for $7 \mathrm{~min}$ in the dark; $8 \mu \mathrm{L}$ of this mixture was then loaded on carbon formvar grids for $5 \mathrm{~min}$ in the dark. Excess solution was absorbed and grids were air-dried for at least $2 \mathrm{~h}$ before observation. Grids were observed with a FEI-TECNAI-Spirit transmission electron microscope (FEI, Hillsboro, Oregon, USA). DLS and TEM were carried out on the nanoparticles before and after the sortase A transpeptidation reaction to assess the effect of the ligation on the physical properties of the nanoparticles.

\section{Sortase A-mediated transpeptidation reaction}

SrtA conjugation reactions were performed in $1 \mathrm{X}$ SrtA reaction buffer $(50 \mathrm{mM}$ Tris- $\mathrm{HCl} \mathrm{pH} 8.0,150 \mathrm{mM}$ $\mathrm{NaCl}, 10 \mathrm{mM} \mathrm{CaCl}$ ). Reactions containing $50 \mu \mathrm{M}$ of SrtA, 50-200 $\mu \mathrm{M}$ of GGG-peptide and $25 \mu \mathrm{M}$ of different PapMV constructions bearing the SrtA recognition motif LPETGG in nanoparticles were incubated for $2.5 \mathrm{~h}$ at room temperature. The molar concentration of nanoparticles was calculated based on the protein concentration of the $\mathrm{CP}$ as determined by $\mathrm{BCA}$ assay, knowing that $1 \mathrm{kDa}=1 \mathrm{~g} / \mathrm{mole}$ and $1 \mu \mathrm{M}=0.000001 \mathrm{~mol} / \mathrm{L}$. Reactions were stopped with the addition of EGTA to a final concentration of $10 \mu \mathrm{M}$. For the PapMV-SrtA-M2e- and PapMV-SrtA-T20-conjugated samples used in the animal studies, unconjugated peptides and SrtA were removed with an amicon centrifugal filter unit with a cutoff size of $100 \mathrm{kDa}$ (EMD Millipore, Darmstadt, Germany). PapMV-SrtA nanoparticles, being larger than $100 \mathrm{kDa}$, were retained by the filter unit. Conjugation efficiency was analyzed by $10 \%$ Tris-Tricine or $15 \%$ Tris-Glycine SDS-PAGE colored with Sypro-Ruby gel stain (Life Technologies Inc. Carlsbad, California, USA). We used 15\% Tris-Glycine SDS-PAGE gels for analysis of the conjugation reaction for better resolution of the PapMV-SrtA and PapMV-SrtA coupled to the peptide (Fig. 3; Additional file 2: Figure S2). SrtA reactions seen in Fig. 3 and Additional file 2: Figure S2 were directly analyzed on SDS-PAGE without eliminating the SrtA enzyme excess peptide by amicon $100 \mathrm{kDa}$.

\section{SDS-PAGE analysis for peptide coupling quantification}

Peptide-labelled PapMV-SrtA nanoparticles were diluted to $0.1 \mu \mathrm{g} / \mu \mathrm{L}$ in cathode buffer for Tris-Tricine gels, or in Tris-Glycine migration buffer supplemented with 30\% of SDS loading buffer (50\% glycerol, 2\% SDS, 0.002\% bromophenol blue, 14\% 2-mercaptoethanol). Samples were then heated at $95^{\circ} \mathrm{C}$ for $10 \mathrm{~min}$ and $4 \mu \mathrm{L}$ of solution $(0.4 \mu \mathrm{g})$ was loaded on $10 \%$ Tris-Tricine SDS-PAGE for the gels shown in Fig. 4 or 15\% Tris/Glycine SDS-Page for the gels shown in Fig. 3 and Additional file 2: Figure S2. Gels were colored with Sypro-Ruby gel stain (Life Technologies Inc. Carlsbad, California, USA) following the manufacturer's rapid staining protocol. Sypro-Ruby fluorescence was detected with the $610 \mathrm{~nm}$ emission filter following excitation using the green laser $(532 \mathrm{~nm})$ of the Typhoon 9200 imager (GE Healthcare Life Sciences). The fluorescence signal resulting from protein bands was quantified using the image analysis software ImageQuant 5.2 , and the intensity volumes associated to the different 
bands, corrected for background signal, were used for the conjugation quantification analysis. The conjugation efficiency was calculated by dividing the signal of coupled PapMV-SrtA protein band by the sum of conjugated and unconjugated PapMV-SrtA signals.

\section{Western blot}

After SDS-PAGE migration, proteins were transferred onto PVDF Immobilon ${ }^{\circledR}$-FL membranes (EMD Millipore, Darmstadt, Germany) and blocked using bløck $^{\text {TM }}$-FL buffer (EMD Millipore). Membranes were incubated overnight with the following primary antibodies diluted in the bløck ${ }^{\mathrm{TM}}$-FL buffer: rabbit anti-PapMV polyclonal antibody, mouse anti-influenza A M2 (14C2) monoclonal antibody (Santa Cruz Biotechnology Inc. Dallas, Texas, USA) or mouse anti-His ${ }_{6}$ polyclonal antibody (Roche, Basel, Switzerland). After washing with PBS/0.1\% Tween-20, membranes were incubated for $2 \mathrm{~h}$ with either Alexa Fluor ${ }^{\circledR} 532$ coupled goat anti-rabbit IgG $(\mathrm{H}+\mathrm{L})$ (Life Technologies Inc. Carlsbad, California, USA) or Alexa Fluor ${ }^{\circledR} 488$ coupled goat anti-mouse IgG $(\mathrm{H}+\mathrm{L})$ (Life Technologies Inc.). After washing, membranes were dried to reduce background, and fluorescent signals were captured using the Typhoon 9200 imager (GE Healthcare Life Sciences).

\section{Animals and immunization}

Six- to 8-week-old female BALB/c mice were immunized by intramuscular injection (i.m.) twice, 2 weeks apart, with buffer $(50 \mu \mathrm{L}$ of $10 \mathrm{mM}$ Tris- $\mathrm{HCl}, \mathrm{pH}$ 8.0) or PapMV-SrtA-M2e, PapMV-sM2e, PapMV-SrtA-T20 or PapMV-SrtA with free T20 peptide. Blood samples were collected before each immunization on days 13 and 27. Serum was separated from the blood by centrifugation in BD Microtainer SST blood collection tubes (BD, East Rutherford, New Jersey, USA) for $2 \mathrm{~min}$ at $10,000 \times g$. Total IgG and IgG2a serotype endpoint titers against M2e and T20 peptides in the sera of immunized mice were determined by enzyme linked immunosorbent assay (ELISA) as described below.

\section{Enzyme linked immunosorbent assay}

M2e (CSLLTEVETPIRNEWGCRCNDSSD) and T20 (GGGYTSLIHSLIEESQNQQEK NEQELLELDKWASLWNWF) peptides were coated overnight at $4{ }^{\circ} \mathrm{C}$ in 96-well flat bottom nunc ${ }^{\mathrm{TM}}$ MaxiSorp plates (VWR, Radnor, Pennsylvania, USA) at 1 and $2.5 \mu \mathrm{g} / \mathrm{mL}$, respectively, in $100 \mu \mathrm{L} 0.1 \mathrm{M} \mathrm{NaHCO}_{3}$ buffer, $\mathrm{pH}$ 9.6. Plates were blocked with $150 \mu \mathrm{L}$ per well of PBS/0.1\% Tween-20/2\% BSA, and washed three times with PBS/0.1\% Tween-20, before adding mice sera in twofold serial dilutions starting at 1:50. Plates were incubated for $1.5 \mathrm{~h}$ at $37{ }^{\circ} \mathrm{C}$, and washed four times in PBS/0.1\% Tween-20 before adding the peroxidase-conjugated goat anti-mouse IgG or IgG2a secondary antibody (Jackson ImmunoResearch Laboratories, West Grove, Pennsylvania, USA). After washing the plates four times, peptide-specific antibodies were detected with the addition of TMB substrate (Fitzgerald Industries International, Acton, Massachusetts, USA). The reaction was stopped with the addition of $0.18 \mathrm{M}$ $\mathrm{H}_{2} \mathrm{SO}_{4}$. Results are expressed as antibody endpoint titers greater than threefold the $\mathrm{OD}_{450 \mathrm{~nm}}$ of the background value of the pre-immune sera at the same dilution.

\section{Influenza challenge}

Two weeks following the last immunization, immunized mice were infected with 1 LD80 of mouse-adapted influenza A/WSN/33 (H1N1). Mice were lightly anesthetized with isoflurane, and infected with $50 \mu \mathrm{L}$ of virus by intranasal instillation. Weight loss and clinical signs of disease were monitored for 14 days post-infection. Symptoms were rated from 0 to 4 as follows: (0) no symptoms: (1) lightly spiked fur and curved back; (2) spiked fur and curved back; (3) difficulty moving and slight dehydration; and (4) severe dehydration, lack of reflexes and ocular secretions. Mice reaching a score of 4 , or having lost $20 \%$ or more of their initial body weight, were euthanized.

\section{Statistical analysis}

Differences (antibody titers, weight and symptoms) between immunized groups were measured by HolmŠídák multiple comparisons test or Tukey's multiple comparisons test. Survival differences were evaluated by Kaplan-Meier survival analysis. $P$-values $<0.05$ were considered statistically significant. Statistical analyses were performed using Graph-Pad Prism version 6.0 (GraphPad Software, La Jolla California USA, http://www.graphpad. com).

\section{Additional files}

Additional file 1: Figure S1. Engineering of PapMV coat protein carrying the SrtA recognition motif missing the linker. (A) The SrtA recognition motif (LPETGG) of SrtA was inserted into the C-terminus of the PapMV coat protein (CP). (B) The size of the VLPS was assessed by dynamic light scattering (DLS). PapMV-SrtA(short) $(65 \mathrm{~nm}$ ) was showed to be slightly longer than the WT PapMV (54 nm). (C) Transmission electron microscopy (TEM) of WT PapMV (left) and PapMV-SrtA(short) (right) nanoparticles.

Additional file 2: Figure S2. Effect of peptide concentrations on the SrtA coupling reaction. (A) Comparison of the C-terminal sequence of PapMV WT, PapMV-SrtA and PapMV-SrtA(short). In PapMV-SrtA, a linker of 5 amino acids (TSTTR) was added before the SrtA recognition motif LPETGG, while in PapMV-SrtA(short) the recongnition motif was included directly in the native PapMV sequence by deleting two proline residues. (B) SDS-PAGE and western blot of SrtA reactions on PapMV-SrtA and PapMV-SrtA(short) nanoparticles. PapMV nanoparticles $(25 \mu \mathrm{M})$ were incubated with SrtA $(50 \mu \mathrm{M})$ and GGG-M2e peptide $(50 \mu \mathrm{M})$ for 2.5 hours at room temperature. Reactions were stopped with EGTA (10 $\mu \mathrm{M})$ and passed through a $100 \mathrm{kDa}$ centrifugal filter unit to eliminate excess 
peptide and contaminating SrtA. PapMV nanoparticles retained by the $100 \mathrm{kDa}$ filter unit were diluted to $0.1 \mathrm{\mu g} / \mathrm{\mu L}$ in migration buffer supplemented with $30 \%$ of SDS loading buffer and $4 \mu \mathrm{L}$ was loaded onto $15 \%$ Tris-Glycine SDS-PAGE. PapMV-SrtA, PapMV-SrtA(short) and SrtA controls correspond to lanes 1, 10 and 11, respectively. Lanes 2-5 and lanes 6-9 represent four experimental replicates of SrtA conjugation on PapMV-SrtA or PapMV-SrtA(short), respectively. Efficient SrtA labelling of GGG-M2e peptide onto PapMV nanoparticles was assessed by SDS-PAGE (top panel), and immunoblotting with a specific antibody against the M2 (bottom panel).

Additional file 3: Figure S3. Optimization of the coupling reaction. SDSPAGE and Western Blots of SrtA reactions in the presence of PapMV-SrtA nanoparticules and increasing concentrations of GGG-M2e peptide. Western blots were directed against the PapMV CP, the $6 \mathrm{xH}$ tag, or the M2e peptide, as indicated on the bottom of each panel. Target products are shown by a red dash. SrtA reactions were diluted to obtain a PapMV-SrtA concentration of $0.1 \mu \mathrm{g} / \mathrm{\mu L}$ (based on molar concentration in the reaction) in migration buffer supplemented with $30 \%$ of SDS loading buffer, and 4 $\mu \mathrm{L}$ was loaded onto $10 \%$ Tris-Tricine SDS-PAGE for analysis.

Additional file 4: Figure S4. PapMV-SrtA-M2e induces a specific antiM2e immune response after a single immunization. Female Balb/C mice, 5 per group, were immunized twice with the indicated formulations. Mice were bled 13 days after the first immunization, and levels of anti-M2e total $\lg G(A)$ and $\lg G 2 a$ (B) were measured by ELISA. ${ }^{* * *} P<0.0001$ for groups 1 , 2, 3 vs 4 for total lgG and lgG2a titers.

Additional file 5: Figure S5. PapMV-SrtA-M2e induces a specific antiM2e immune response and protection against an influenza challenge(repeat). Female Balb/C mice (10 per group) were immunized twice with

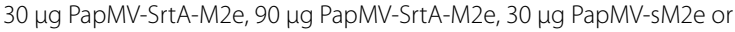
formulation buffer. At 13 days following the last immunization, mice were bled and ELISA assays performed to evaluate levels of anti-M2e total IgG (A) or $\lg \mathrm{G} 2 \mathrm{a}(\mathrm{B}) .{ }^{* * * *} \mathrm{P}<0.0001$ for groups 1, 2, 3 vs 4 total IgG titers and IgG2a titers. Mice were infected with 1 x LD80 of influenza A/WSN/33 virus 14 days after the last immunization, and followed for clinical symptoms and survival for 14 days. (C) Mean weight loss expressed as percentage of initial weight. ${ }^{* *}<<0.01$ for group 1 vs $2,{ }^{* * *} P<0.001$ for group 3 vs 4 , and for group 2 vs $3,{ }^{* * * *} P<0.0001$ for group 2 vs 4 , all at day 7 post-challenge. (D) Mean clinical score of infection signs on a scale of 0 to 4 . ${ }^{* * *} P<0.001$ for group 1 vs 3 , and ${ }^{* * * *} P<0.0001$ for group 1 vs 2 and groups 2,3 vs 4 . (E) Survival of mice expressed as Kaplan-Meier survival curves. ${ }^{*} P<0.5$ for groups 2 vs 4 and ${ }^{* *} P<0.01$ for group 3 vs 4 .

\section{Authors' contributions}

AT performed the final PapMV-SrtA construct screening, the coupling experiments on the PapMV platform, and was involved with experiments with animals. AT also drafted the manuscript. MB performed the structural model of PapMV and helped draft the manuscript. DC and GR helped perform mouse experiments, and to draft the manuscript. LGL contributed to the initial screening of PapMV constructs harbouring the SrtA recognition motif. MB, MELG and PS were responsible for production of batches of PapMV nanoparticles used in this work. DL supervised the entire study, coordinated the involvement of each lab member and finalised the manuscript. All authors read and approved the final manuscript.

\section{Author details}

1 Department of Microbiology, Infectiology and Immunology, Infectious Disease Research Center, Laval University, 2705 Boul. Laurier, Quebec City, PQ GIV 4G2, Canada. ${ }^{2}$ Neurosciences, Laval University, 2705 Boul. Laurier, Québec City, PQ G1V 4G2, Canada.

\section{Acknowledgements}

We would like to thank the "Plateforme de bio-imagerie du Centre de Recherche en Infectiologie"for the use of the transmission electron microscope. Also, we would like to thank Helen Rothnie for her help in editing the manuscript.

\section{Competing interests}

Authors Denis Leclerc and Pierre Savard are shareholders of the company FOLIA BIOTECH INC., a start-up company that has the mandate to exploit this technology commercially to improve currently available vaccines and create new vaccines. This does not alter the authors' adherence to all the journal policies.

\section{Ethics statement}

All animal work was performed in accordance with the institution-approved ethics protocol of the "Comité de Protection des Animaux - CHUQ (CPA(HUQ)". The approval for this project can be found under Authorization Number 2013142-2.

\section{Funding}

We would like to thank the Natural Sciences and Engineering Research Council of Canada (NSERC Grant RGPIN-2016-05852) and the Canadian Institute of Health Research (CIHR Grant 298143) for funding this research program.

\section{Publisher's Note}

Springer Nature remains neutral with regard to jurisdictional claims in published maps and institutional affiliations.

Received: 5 October 2016 Accepted: 10 July 2017

Published online: 18 July 2017

\section{References}

1. Chroboczek J, Szurgot I, Szolajska E. Virus-like particles as vaccine. Acta Biochim Pol. 2014;61(3):531-9.

2. Kushnir N, Streatfield SJ, Yusibov V. Virus-like particles as a highly efficient vaccine platform: diversity of targets and production systems and advances in clinical development. Vaccine. 2012;31(1):58-83.

3. Lee KL, Twyman RM, Fiering S, Steinmetz NF. Virus-based nanoparticles as platform technologies for modern vaccines. WIREs Nanomed Nanobiotechnol. 2016;8(4):554-78.

4. Plummer EM, Manchester M. Viral nanoparticles and virus-like particles: platforms for contemporary vaccine design. WIREs Nanomed Nanobiotechnol. 2011;3(2):174-96.

5. Lua LHL, Connors NK, Sainsbury F, Chuan YP, Wibowo N, Middelberg APJ. Bioengineering virus-like particles as vaccines. Biotechnol Bioeng. 2014;111(3):425-40.

6. Noad R, Roy P. Virus-like particles as immunogens. Trends Microbiol. 2003;11(9):438-44.

7. Rodríguez-Limas WA, Sekar K, Tyo KE. Virus-like particles: the future of microbial factories and cell-free systems as platforms for vaccine development. Curr Opin Biotechnol. 2013;24(6):1089-93.

8. Zeltins A. Construction and characterization of virus-like particles. Mol Biotechnol. 2013;53(1):92-107.

9. Zhao L, Seth A, Wibowo N, Zhao CX, Mitter N, Yu C, et al. Nanoparticle vaccines. Vaccine. 2014;32(3):327-37.

10. Roldão A, Mellado MCM, Castilho LR, Carrondo MJT, Alves PM. Virus-like particles in vaccine development. Expert Rev Vaccines. 2010;9(10):1149-76.

11. Fifis T, Gamvrellis A, Crimeen-Irwin B, Pietersz GA, Li J, Mottram PL, et al. Size-dependent immunogenicity: therapeutic and protective properties of nano-vaccines against tumors. J Immunol. 2004;173(5):3148-54.

12. Manolova V, Flace A, Bauer M, Schwarz K, Saudan P, Bachmann MF. Nanoparticles target distinct dendritic cell populations according to their size. Eur J Immunol. 2008;38(5):1404-13.

13. Lebel M-E, Chartrand K, Leclerc D, Lamarre A. Plant viruses as nanoparticle-based vaccines and adjuvants. Vaccines. 2015;3(3):620-37.

14. Mathieu C, Rioux G, Dumas MCC, Leclerc D. Induction of innate immunity in lungs with virus-like nanoparticles leads to protection against influenza and Streptococcus pneumoniae challenge. Nanomedicine. 2013;9(7):839-48.

15. Lebel M-E, Daudelin J-F, Chartrand K, Tarrab E, Kalinke U, Savard P, Leclerc D, Lamarre A. Nanoparticle adjuvant sensing by TLR7 enhances CD8+ T 
cell-mediated protection from Listeria monocytogenes infection. J Immunol. 2014;192(3):1071-8.

16. Savard C, Guérin A, Drouin K, Bolduc M, Laliberté-Gagné M-E, Dumas M-C, Majeau N, Leclerc D. Improvement of the trivalent inactivated flu vaccine using PapMV nanoparticles. PLoS ONE. 2011;6(6):e21522.

17. Lebel M-E, Chartrand K, Tarrab E, Savard P, Leclerc D, Lamarre A. Potentiating cancer immunotherapy using papaya mosaic virus-derived nanoparticles. Nano Lett. 2016;16(3):1826-32.

18. Leclerc D. Plant viral epitope display systems for vaccine development. Curr Top Microbiol Immunol. 2014;375:47-59.

19. Carignan D, Thérien A, Rioux G, Paquet G, Gagné MĖL, Bolduc M, Savard P, Leclerc D. Engineering of the PapMV vaccine platform with a shortened M2e peptide leads to an effective one dose influenza vaccine. Vaccine. 2015;33(51):7245-53.

20. Rioux G, Babin C, Majeau N, Leclerc D. Engineering of papaya mosaic virus (papmv) nanoparticles through fusion of the HA11 peptide to several putative surface-exposed sites. PLoS ONE. 2012;7(2):5-12.

21. Babin C, Majeau N, Leclerc D. Engineering of papaya mosaic virus PapMV nanoparticles with a CTL epitope derived from influenza NP. J Nanobiotechnol. 2013;11:10

22. Leclerc D, Beauseigle D, Denis J, Morin H, Pare C, Lamarre A, Lapointe R. Proteasome-independent MHC class I cross-presentation mediated by papaya mosaic virus-like particles leads to the expansion of specific human T cells. J Virol. 2007;81(3):1319-26.

23. Lacasse P, Denis J, Lapointe R, Leclerc D, Lamarre A. Novel plant virusbased vaccine induces protective CTL-mediated antiviral immunity through dendritic cell maturation. J Virol. 2008;82(2):785-94.

24. Hanafi LA, Bolduc M, Laliberté-Gagné ME, Dufour F, Langelier Y, Boulassel MR, Routy JP, Leclerc D, Lapointe R. Two distinct chimeric potexviruses share antigenic cross-presentation properties of MHC class I epitopes. Vaccine. 2010;28(34):5617-26.

25. Clancy KW, Melvin J, McCafferty DG. Sortase transpeptidases: insights into mechanism, substrate specificity, and inhibition. Biopolymers. 2010;94(4):385-96.

26. Ritzefeld M. Sortagging: a robust and efficient chemoenzymatic ligation strategy. Chemistry. 2014;20(28):8516-29.

27. Spirig T, Weiner EM, Clubb RT. Sortase enzymes in Gram-positive bacteria. Mol Microbiol. 2011;82(5):1044-59.

28. Schoonen L, Pille J, Borrmann A, Nolte RJ, van Hest JC. Sortase A-mediated $\mathrm{N}$-terminal modification of cowpea chlorotic mottle virus for highly efficient cargo loading. Bioconjug Chem. 2015;26(12):2429-34.

29. Jiang R, Wang L, Weingart J, Sun XLL. Chemoenzymatic bio-orthogonal chemistry for site-specific double modification of recombinant thrombomodulin. ChemBioChem. 2014:15(1):42-6.

30. Matsumoto T, Sawamoto S, Sakamoto T, Tanaka T, Fukuda H, Kondo A. Site-specific tetrameric streptavidin-protein conjugation using sortase A. J Biotechnol. 2011;152(1-2):37-42.

31. Parthasarathy R, Subramanian S, Boder ET. Sortase A as a novel molecular "stapler" for sequence-specific protein conjugation. Bioconjug Chem. 2007;18(2):469-76.

32. Steinhagen M, Zunker K, Nordsieck K, Beck-Sickinger AG. Large scale modification of biomolecules using immobilized sortase A from Staphylococcus aureus. Bioorganic amp Med Chem. 2013;21(12):3504-10.

33. Sijbrandij T, Cukkemane N, Nazmi K, Veerman EC, Bikker FJ. Sortase A as a tool to functionalize surfaces. Bioconjug Chem. 2013;24(5):828-31.

34. Pritz $\mathrm{S}$, Wolf Y, Kraetke $\mathrm{O}$, Klose J, Bienert M, Beyermann M. Synthesis of biologically active peptide nucleic acid — peptide conjugates by sortasemediated ligation. J Org Chem. 2007;72(10):3909-12.

35. Antos JM, Miller GM, Grotenbreg GM, Ploegh HL. Lipid modification of proteins through sortase-catalyzed transpeptidation. J Am Chem Soc. 2008;130(48):16338-43.

36. Samantaray S, Marathe U, Dasgupta S, Nandicoori VK, Roy RP. Peptidesugar ligation catalyzed by transpeptidase sortase: a facile approach to neoglycoconjugate synthesis. J Am Chem Soc. 2008;130(7):2132-3.

37. Levary DA, Parthasarathy R, Boder ET, Ackerman ME. Protein-protein fusion catalyzed by sortase A. PLOS ONE. 2011:6(4):e18342.
38. Madej MP, Coia G, Williams CC, Caine JM, Pearce LA, Attwood R, et al. Engineering of an anti-epidermal growth factor receptor antibody to single chain format and labeling by sortase A-mediated protein ligation. Biotechnol Bioeng. 2012;109(6):1461-70.

39. DiMaio F, Chen C-C, Yu X, Frenz B, Hsu Y-H, Lin N-S, et al. The molecular basis for flexibility in the flexible filamentous plant viruses. Nat Struct Mol Biol. 2015;22(8):642-4.

40. Agirrezabala X, Méndez-López E, Lasso G, Sánchez-Pina MA, Aranda M, Valle $M$. The near-atomic cryoEM structure of a flexible filamentous plant virus shows homology of its coat protein with nucleoproteins of animal viruses. Elife. 2015:4:e11795.

41. Yang S, Wang T, Bohon J, Gagné ME, Bolduc M, Leclerc D, Li H. Crystal structure of the coat protein of the flexible filamentous papaya mosaic virus. J Mol Biol. 2012;422(2):263-73.

42. Yang J, Yan R, Roy A, Xu D, Poisson J, Zhang Y. The I-TASSER Suite: protein structure and function prediction. Nat Methods. 2015;12(1):7-8.

43. Neirynck S, Deroo T, Saelens $X$, Vanlandschoot $P$, Jou WM, Fiers W. A universal influenza A vaccine based on the extracellular domain of the M2 protein. Nat Med. 1999;5(10):1157-63.

44. Denis J, Acosta-Ramirez E, Zhao Y, Hamelin ME, Koukavica I, Baz M, et al. Development of a universal influenza A vaccine based on the M2e peptide fused to the papaya mosaic virus (PapMV) vaccine platform. Vaccine. 2008;26(27-28):3395-403.

45. He Y. Synthesized peptide inhibitors of HIV-1 gp41-dependent membrane fusion. Curr Pharm Des. 2013;19(10):1800-9.

46. Serrano S, Araujo A, Apellániz B, Bryson S, Carravilla P, De La Arada I. Structure and immunogenicity of a peptide vaccine, including the complete HIV-1 gp41 2F5 epitope: implications for antibody recognition mechanism and immunogen design. J Biol Chem. 2014;289(10):6565-80.

47. Rioux G, Mathieu C, Russell A, Bolduc M, Laliberté-Gagné M-E, Savard P, et al. PapMV nanoparticles improve mucosal immune responses to the trivalent inactivated flu vaccine. J Nanobiotechnol. 2014;12:19.

48. Denis J, Majeau N, Elizabeth A-R, Savard C, Bedard MCC, Simard S, et al. Immunogenicity of papaya mosaic virus-like particles fused to a hepatitis C virus epitope: evidence for the critical function of multimerization. Virology. 2007;363(1):59-68.

49. Hess GT, Cragnolini JJ, Popp MW, Allen MA, Dougan SK, Spooner E, et al. M13 bacteriophage display framework that allows sortase-mediated modification of surface-accessible phage proteins. Bioconjug Chem. 2012;23(7):1478-87.

50. Tang $S$, Xuan B, Ye X, Huang Z, Qian Z. A modular vaccine development platform based on sortase-mediated site-specific tagging of antigens onto virus-like particles. Sci Rep. 2016;12(6):25741.

51. Gamvrellis A, Leong D, Hanley JC, Xiang SD, Mottram P, Plebanski M. Vaccines that facilitate antigen entry into dendritic cells. Immunol Cell Biol. 2004;82(5):506-16.

52. Jegerlehner A, Schmitz N, Storni T, Bachmann MF. Influenza A vaccine based on the extracellular domain of M2: weak protection mediated via antibody-dependent NK cell activity. J Immunol. 2004;172(9):5598-605.

53. Popp MW, Antos JM, Ploegh HL. Site-specific protein labeling via sortasemediated transpeptidation. Curr Protoc Protein Sci. 2009;15:Unit 15.3.

\section{Submit your next manuscript to BioMed Central and we will help you at every step:}

- We accept pre-submission inquiries

- Our selector tool helps you to find the most relevant journal

- We provide round the clock customer support

- Convenient online submission

- Thorough peer review

- Inclusion in PubMed and all major indexing services

- Maximum visibility for your research

Submit your manuscript at www.biomedcentral.com/submit 